\title{
Structure and Properties of Hydrogen-Impurity Pairs in Elemental Semiconductors
}

\author{
P. J. H. Denteneer, ${ }^{(a)}$ C. G. Van de Walle, ${ }^{(b)}$ and S. T. Pantelides \\ IBM Research Division, Thomas J. Watson Research Center, Yorktown Heights, New York 10598 \\ (Received 3 January 1989)
}

\begin{abstract}
A variety of experiments have revealed several puzzling properties of hydrogen-impurity pairs. For example, $\mathrm{H}$ atoms passivate the electrical activity of some impurities, whereas they induce electrical activity in others; they appear to tunnel around some impurities but not around others. We report firstprinciples pseudopotential-density-functional calculations for several hydrogen-impurity complexes and unravel the origins and intricacies of the rich behavior of $\mathrm{H}$ bound to different substitutional impurities in $\mathrm{Si}$ and $\mathrm{Ge}$.

PACS numbers: $61.70 . \mathrm{Bv}, 66.30 . \mathrm{Jt}, 71.55 . \mathrm{Ht}$
\end{abstract}

Over the years experimental observations have unveiled a very diverse role for hydrogen atoms in semiconductors containing impurities. In virtually all cases, $\mathrm{H}$ atoms are found to form pairs with substitutional impurities but their effect on electrical activity has been puzzling. ${ }^{1-7}$ In some cases, as for example substitutional boron or phosphorus in $\mathrm{Si}, \mathrm{H}$ passivates the electrical activity of the impurity. ${ }^{4-7}$ In other cases, as for example substitutional $\mathrm{Si}$ in $\mathrm{Ge}, \mathrm{H}$ converts a normally inactive impurity into a shallow acceptor. ${ }^{2,3}$ Alternatively, this amphoteric effect of $\mathrm{H}$ on the electrical activity of impurities can be described ${ }^{3}$ by stating that sometimes the complex behaves as a substitutional atom that lies one column to the left of the impurity in the Periodic Table, e.g., the $(\mathrm{H}, \mathrm{Si})$ complex in ultrapure $\mathrm{Ge}$, whereas in other cases the complex behaves as a substitutional atom on column to the right of the impurity in the Periodic Table, e.g., the $(\mathrm{H}, \mathrm{B})$ complex in $\mathrm{Si}$. Suggestions for the origins of this unusual behavior have been made on the basis of semiempirical calculations, ${ }^{8}$ but the conclusions were only tentative.

A second question that has been debated extensively over the years is whether $\mathrm{H}$ is tunneling around the impurity as opposed to occupying a particular site close to the impurity. For example, certain experimental evidence led to the belief that $\mathrm{H}$ tunnels around $\mathrm{Si}$ and $\mathrm{C}$ in $\mathrm{Ge},{ }^{2}$ but subsequent experiments showed that a static model with trigonal symmetry was more appropriate for the acceptor complexes. ${ }^{3}$ In contrast, Muro and Sievers ${ }^{9}$ found evidence of tunneling hydrogen in the hydrogenberyllium acceptor complex in $\mathrm{Si}$. The experimental findings were satisfactorily accounted for by the dynamic tunneling model of Ref. 2. On the other hand, there is no evidence that $\mathrm{H}$ tunnels around $\mathrm{Be}$ in $\mathrm{Ge}$. No theoretical understanding of the conditions that favor tunneling is available.

A third question that attracted considerable attention is the specific atomic configuration of $\mathrm{H}$-impurity pairs. Most of the attention so far has focused on the $(\mathrm{H}, \mathrm{B})$ pair in Si. A large number of theoretical calculations has been reported contrasting the properties of only a few configurations. ${ }^{10-13}$ Though the configuration hav- ing $\mathrm{H}$ in one of the four $\mathrm{Si}-\mathrm{B}$ bonds is favored on the basis of total-energy calculations, the results are not definitive because no search has been made for the global total-energy minimum with full relaxation of the host crystal. Also, it is generally believed that the $(\mathrm{H}, \mathrm{Be})$ complex in $\mathrm{Si}$ consists of an $\mathrm{H}$ atom tunneling around $\mathrm{Be}$ between four equivalent antibonding $(A B)$ sites on the extension of $\mathrm{Si}-\mathrm{Be}$ bonds. There is no experimental or theoretical evidence, however, that establishes this over other possible paths.

All of the above questions regarding the interaction of $H$ with substitutional impurities in semiconductors can be addressed simultaneously by calculating the totalenergy surfaces for an $\mathrm{H}$ atom around each specific impurity and by a concomitant examination of the corresponding energy levels in the energy gap. In this Letter we report the results of such a study for three qualitatively different hydrogen-impurity complexes. The main conclusions are as follows: Acceptor impurities such as $\mathrm{B}$ or $\mathrm{Be}$ bind an $\mathrm{H}$ atom rather strongly at several symmetrically equivalent sites in their immediate vicinity. Barriers for $\mathrm{H}$ motion around the impurity between such sites are small by comparison with the binding energy, so that motion around the impurity can occur either thermally or quantum mechanically (tunneling), depending on subtle differences between the complexes. In contrast, isovalent impurities, such as $\mathrm{Si}$ in $\mathrm{Ge}$, bind an $\mathrm{H}$ atom very weakly, and the barrier for possible motion around the impurity is significantly larger than the binding energy so that the resulting pairs are static. The effect of $\mathbf{H}$ on the electrical activity of the impurity in each case follows naturally from the bonding properties of the complexes.

The calculations are carried out using the firstprinciples pseudopotential-density-functional method. The method is well documented ${ }^{14}$ and has been shown to accurately reproduce and predict ground-state properties of semiconductors. Its successful application to defects and defect complexes is documented in Refs. 15-17. We use periodically repeated supercells to describe the host crystal (including the substitutional impurity) in which $\mathrm{H}$ resides. In order to include all relevant relaxations of 


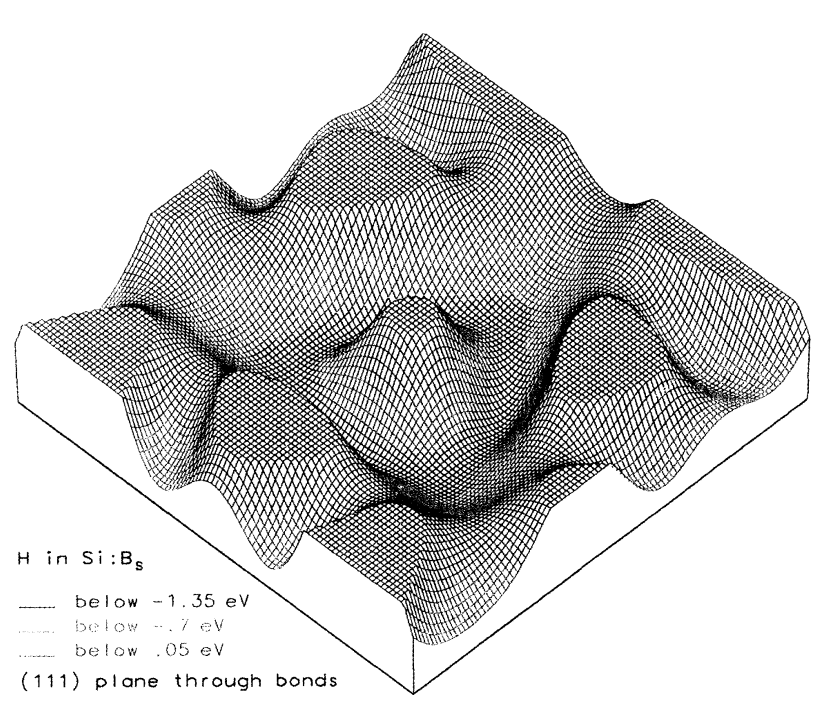

FIG. 1. Energy surface for an $\mathrm{H}$ atom in the (111) plane through three bond-minima $(B M)$ positions in $\mathrm{Si} \mathrm{B}_{s}$. The plane does not contain atoms, but the unrelaxed lattice position of the $\mathrm{B}$ atom is located just $0.4 \AA$ outside the plane in the center of the red ring. The contours are color coded in three different ranges for presentation purposes. For clarity, the surface is cut off at an energy value of $0.05 \mathrm{eV}$, resulting in the green plateaus. The zero of energy is chosen at the tetrahedral interstitial site.

the host crystal for all of the $\mathrm{H}$ positions considered it is necessary to use supercells of up to 32 atoms. ${ }^{18} \mathrm{We}$ find that most properties of the complexes are described accurately when we use expansions of the wave functions in plane waves with kinetic energy up to $12 \mathrm{Ry} .{ }^{19}$ In order to calculate energy barriers with an accuracy of $\lesssim 0.1$ $\mathrm{eV}$, kinetic energy cutoffs of up to $20 \mathrm{Ry}$ in 32 -atom cells are used. Two to four special $\mathbf{k}$ points (depending on the symmetry of the $\mathbf{H}$ position) are used to integrate over the first Brillouin zone of the 32-atom cell, which is found to induce negligible error bars on calculated energy differences. Complete energy surfaces for an $\mathrm{H}$ atom in the neighborhood of a substitutional impurity in either $\mathrm{Si}$ or Ge are obtained by making use of the symmetry of the host crystal. ${ }^{16,18}$

The main result of our calculations is that both $(\mathrm{H}, \mathrm{B})$ and $(\mathrm{H}, \mathrm{Be})$ in $\mathrm{Si}$ exhibit a low-energy shell around the impurity, primarily going through sites close to the center of a Si-impurity bond (bond minimum or $B M$ site) and sites labeled $C$ (midway between any two of the impurity's nearest neighbors). The low-energy shell is clearly visible as a ring in the total-energy surface for an $\mathrm{H}$ atom in the (111) plane shown in Fig. 1. In the contour plot of Fig. 2 for $\mathrm{H}$ in the (110) plane only half a ring containing the $B M$ and $C$ sites is visible. The lower part of Fig. 2 contains antibonding sites $(A B)$, which are clearly saddle points, another $C$ site, and the tetrahedral interstitial site $\left(T_{d}\right)$, which is a local maximum. The $A B$

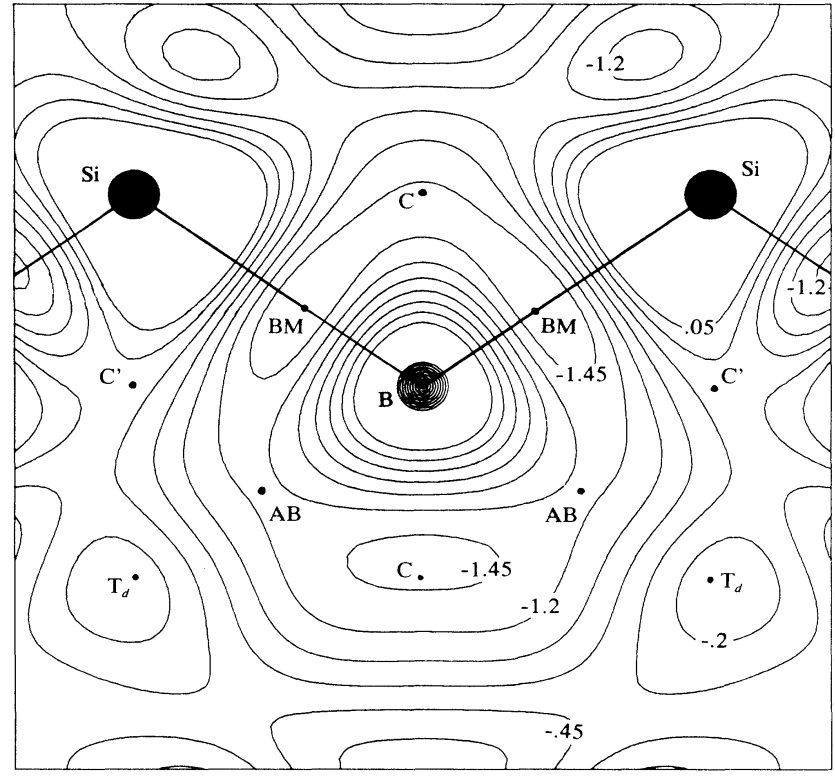

FIG. 2. Contour plot of the energy surface for an $\mathrm{H}$ atom in the (110) plane in $\mathrm{Si}: \mathrm{B}_{s}$. Big dots indicate (unrelaxed) atomic positions; bonded atoms are connected by solid lines. The substitutional boron atom occupies the center of the plot. Positions of special interest are indicated (see text). Sites denoted $C$ and $C^{\prime}$ are equivalent if the $\mathrm{B}$ atom in the middle is replaced by a $\mathrm{Si}$ atom. The unit of energy is $\mathrm{eV}$ and the spacing between contours is $0.25 \mathrm{eV}$. Close to the atoms contours are not shown above an energy value of $0.05 \mathrm{eV}$. All relevant relaxations are taken into account to calculate total energies, but the relaxations of the host-crystal atoms are not shown in the figure because they are different for different positions of $\mathrm{H}$.

site is $0.5 \mathrm{eV}$ higher in energy than the $B M$ site and can only be mistaken for a minimum if only sites for $\mathrm{H}$ along the $\langle 111\rangle$ direction are considered. ${ }^{11}$ The result that the $A B$ site is a saddle point definitively rules out as the stable site for $\mathrm{H}$.

The energy surface for $(\mathrm{H}, \mathrm{Be})$ in $\mathrm{Si}$ is qualitatively the same as for $(\mathrm{H}, \mathrm{B})$ in $\mathrm{Si}$. In each case a low-energy path through $B M$ and $C$ sites is available. In the case of $(\mathrm{H}, \mathrm{B})$ the $B M$ site is the global minimum with a site close to $C$ being the saddle point for motion of $\mathrm{H}$, whereas in the case of $(\mathrm{H}, \mathrm{Be})$ the roles of $B M$ and $C$ are reversed. More specifically, for $(H, B)$ the saddle point is $0.2 \mathrm{eV}$ higher in energy than the $B M$ site, whereas for $(\mathrm{H}, \mathrm{Be})$ the $C$ site is $0.1 \mathrm{eV}$ lower than the $B M$ site. For $(\mathrm{H}, \mathrm{Be})$ the $A B$ site is $0.4 \mathrm{eV}$ higher than the $C$ site and again a saddle point. In the lowest-energy $(B M)$ configuration for $(\mathrm{H}, \mathrm{B})$ the $\mathrm{Si}$ and $\mathrm{B}$ atoms closest to $\mathrm{H}$ relax outward by the large amounts of 0.24 and $0.42 \AA$, respectively. Second-nearest-neighbor relaxations are also significant in this configuration. In contrast, the lowest-energy ( $C$ site) configuration for the $(\mathrm{H}, \mathrm{Be})$ complex only involves a small relaxation of $\mathrm{Be}$ of $0.14 \AA$ 
away from $\mathrm{H}$.

The $B M$ configuration for $(\mathrm{H}, \mathrm{B})$ is in agreement with a wealth of experimental observations, ${ }^{20-23}$ although sometimes a slightly off-axis position close to the bond center is proposed for $\mathrm{H}^{22}$ Also the majority of theoretical calculations appear to agree now on a configuration similar to the $B M$ configuration. ${ }^{10,12,13}$ Furthermore, our calculated vibrational frequency of the $H$ stretching mode for the $B M$ configuration of $1830 \pm 100 \mathrm{~cm}^{-1}$ is in good agreement with the experimental value ${ }^{21}$ of 1903 $\mathrm{cm}^{-1}$. Similar experimental information for the $(\mathrm{H}, \mathrm{Be})$ complex is presently not available, but since all of the features of the microscopic structure of the $(\mathrm{H}, \mathrm{B}) \mathrm{com}$ plex are in excellent agreement with experimental observations, we can be confident of our description of the $(\mathrm{H}, \mathrm{Be})$ complex.

In contrast to the case of $(\mathrm{H}, \mathrm{B})$ and $(\mathrm{H}, \mathrm{Be})$ in $\mathrm{Si}$, where we find a low-energy region surrounding the impurity, in the case of $(\mathrm{H}, \mathrm{Si})$ in Ge the total-energy surfaces of $\mathrm{H}$ in various charge states are virtually identical to the surfaces one obtains in the pure material without low-energy regions restricted to the neighborhood of the impurity. ${ }^{16}$ This is to be expected since $\mathrm{Si}$ and Ge are very similar. For the three charge states considered (positive, neutral, and negative) the global energy minima for $\mathrm{H}$ in $\mathrm{Ge}: \mathrm{Si}_{s}$ are the bond-centered site for $\mathrm{H}^{+}$ and $\mathrm{H}^{0}$, and a site close to the $T_{d}$ site (displaced from $T_{d}$ over $0.2 \AA$ toward $\mathrm{Si}$ ) for $\mathrm{H}^{-}$. Although $\mathrm{Si}$ and $\mathrm{Ge}$ are very similar and one would not expect the isovalent impurity $\mathrm{Si}$ in $\mathrm{Ge}$ to be able to bind $\mathrm{H}$, the $(\mathrm{H}, \mathrm{Si})$ complex in Ge has a positive binding energy. ${ }^{24}$ The binding energies for the three minimum configurations turn out to be very small, but consistently positive (i.e., the complex is bound); we find $E_{b}=20,28$, and $52 \mathrm{meV}$ for $\mathrm{H}^{+}$, $\mathbf{H}^{0}$, and $\mathrm{H}^{-}$, respectively. Since barriers for movement of $\mathrm{H}$ around the $\mathrm{Si}$ impurity are much larger than these binding energies (e.g., for $\mathrm{H}^{-}$there is a saddle point for possible motion of $\mathrm{H}$ at the hexagonal interstitial site with a barrier of $0.35 \mathrm{eV}$ ), the $\mathrm{H}$ atom cannot move around the Si impurity while still being bound. We will return to the question of motion of $\mathrm{H}$ around impurities later on in the paper.

Regarding the effect of the $\mathrm{H}$ atom on the electrical activity of substitutional impurities, we arrive at the surprising result that in all cases the $\mathrm{H}$-impurity pair has an energy level that is virtually identical to the level of an $\mathrm{H}$ atom at the same site without the impurity. Whether the impurity is deactivated or activated by $\mathrm{H}$ is merely a consequence of the specific site that $\mathrm{H}$ occupies near the impurity. In the case of $\mathrm{B}$ and $\mathrm{Be}, \mathrm{H}$ is located in the region close to the impurity (containing $B M$ and $C$ sites). For such positions the $\mathrm{H}$-related level occurs at midgap. ${ }^{16}$ The electron of $\mathbf{H}$ drops in the empty acceptor level and reduces the activity of the impurity by one unit: The $(H, B)$ complex is completely inactive and the $(\mathrm{H}, \mathrm{Be})$ complex is a single acceptor. For the $(\mathrm{H}, \mathrm{Si})$ complex in $\mathrm{Ge}$ the influence of $\mathrm{H}$ on electrical activity depends on the Fermi-level position, since the Fermilevel position determines which charge state and site are favored. We find that for $p$-type Ge (Fermi level close to the top of the valence bands) $\mathrm{H}^{+}$is $0.2 \mathrm{eV}$ lower in energy than $\mathrm{H}^{-}$, which is $0.2 \mathrm{eV}$ lower in energy than $\mathrm{H}^{0}$. Therefore, in $p$-type $\mathrm{Ge}, \mathrm{H}$ acts as a donor, just like in $p$-type $\mathrm{Si}^{16}$ As a consequence a $(\mathrm{H}, \mathrm{Si})$ complex in $p$ type Ge would behave as a donor (this is, of course, a hypothetic case since $\mathrm{H}$ would first pair with the acceptors before pairing with isovalent $\mathrm{Si}$ impurities). In $n$-type $\mathrm{Ge}, \mathrm{H}^{-}$close to $T_{d}$ is the lowest-energy state. In ultrapure $\mathrm{Ge}$, in which $(\mathrm{H}, \mathrm{Si})$ complexes have been observed, the Fermi level is effectively located in the middle of the gap. In that case, $\mathrm{H}^{-}$close to $T_{d}$ is the lowest-energy state. For a position of $\mathrm{H}$ close to $T_{d}$ an $\mathrm{H}$-related level is found below the top of the valence bands. The level will be doubly occupied leaving a hole in the top of the valence band. Therefore, the $(\mathrm{H}, \mathrm{Si})$ complex with $\mathrm{H}$ close to the $T_{d}$ site acts as an acceptor in agreement with the experimental observation in ultrapure Ge. ${ }^{2}$

We now turn to the question of motion of the $\mathrm{H}$ atom in $\mathrm{H}$-impurity pairs. As we saw above, in the $(\mathrm{H}, \mathrm{Si})$ complex in $\mathrm{Ge}, \mathrm{H}$ cannot move around the impurity since the binding energy of $(\mathrm{H}, \mathrm{Si})$ is much smaller than any barrier $\mathrm{H}$ would have to overcome. However, in both the cases of $(\mathrm{H}, \mathrm{B})$ and $(\mathrm{H}, \mathrm{Be})$ in $\mathrm{Si}$, the $\mathrm{H}$ atom is firmly bound with a binding energy of about $1 \mathrm{eV}$ (referenced with respect to a dissociated state of isolated ionized acceptors and neutral $\mathrm{H}$ atoms in $\mathrm{Si}$ and with the Fermi level close to the top of the valence bands). From the energy surfaces discussed above we already saw that barriers for motion of $\mathrm{H}$ around the impurity are small: $0.2 \mathrm{eV}$ for $(\mathrm{H}, \mathrm{B})$ and $0.1 \mathrm{eV}$ for $(\mathrm{H}, \mathrm{Be})$. Such barriers can easily be overcome when $\mathbf{H}$ is moving thermally. Very recently, in experiments using the optical dichroism of the H-B absorption bands under uniaxial stress, an activation energy of $0.19 \mathrm{eV}$ was found for $\mathrm{H}$ motion from one $B M$ site to another, in agreement with our calculated result. $^{23}$

We now consider the possibility that $\mathrm{H}$ would tunnel around the substitutional impurity. Such tunneling may occur because of the small mass of the $\mathrm{H}$ atom. The much heavier $\mathrm{Si}$ or impurity atoms do not participate in the quantum-mechanical process, and merely define the potential in which the light particle moves. These potential wells should be calculated by keeping the host crystal atoms fixed at the positions they have for the initial lowest-energy configuration. For tunneling to occur, the resulting potential must have two or more identical or similar wells separated by small barriers. ${ }^{25}$

In the case of $(\mathrm{H}, \mathrm{Be})$, the global minimum is at the $C$ site, with little relaxation of the host. Tunneling between equivalent $C$ sites can occur if there is a path that does not require motion of the host atoms and the corresponding barrier is small. Tunneling through the $B M$ site is not possible because, with the host atoms frozen, we find a barrier of $2.4 \mathrm{eV}$. We have, however, identified a tun- 
neling path going through an $A B$ site, with a barrier of $0.4 \mathrm{eV}$. An estimate of tunneling frequencies in a onedimensional model has shown that such a barrier is consistent with the possibility of tunneling in this system.

In the case of $(\mathrm{H}, \mathrm{B})$, the global minimum is at the $B M$ site, which requires large relaxations of the neighboring $\mathrm{B}$ and $\mathrm{Si}$ atoms. With $\mathrm{H}$ located at one bond center, these relaxations are such that the adjacent bond centers are high in energy and thus do not provide a potential well for the $\mathbf{H}$ atom to tunnel to. Thus, tunneling between equivalent $B M$ sites is not possible.

In conclusion, our theoretical calculations reveal that $H$ occupies different sites when it pairs with different impurities, and that the nature of the site determines both the electrical activity of the pair and the possibility of thermal and quantum-mechanical motion around the impurity.

This work was supported in part by the U.S. Office of Naval Research under Contract No. N00014-84-C0396. One of us (P.J.H.D.) acknowledges support from IBM Netherlands, N.V.

(a) Present address: Physics Department, University of Nijmegen, Toernooiveld 1, 6525 ED Nijmegen, The Netherlands.

(b) Present address: Philips Laboratories, 345 Scarborough Road, Briarcliff Manor, NY 10510.

${ }^{1}$ E. E. Haller, in Proceedings of the Third International Conference on Shallow Impurities in Semiconductors, Linköping, Sweden, 1988 (to be published).

${ }^{2}$ E. E. Haller, B. Joos, and L. M. Falicov, Phys. Rev. B 21, 4729 (1980).

${ }^{3}$ J. M. Kahn, R. E. McMurray, Jr., E. E. Haller, and L. M. Falicov, Phys. Rev. B 36, 8001 (1987).

${ }^{4}$ C. T. Sah, J. Y. C. Sun, and J. J. T. Tzou, Appl. Phys. Lett. 43, 204 (1983).

${ }^{5}$ K. Bergman, M. Stavola, S. J. Pearton, and J. Lopata, Phys. Rev. B 37, 2770 (1988).

${ }^{6}$ J. I. Pankove, D. E. Carlson, J. E. Berkeyheiser, and R. O. Wance, Phys. Rev. Lett. 51, 2224 (1983).

${ }^{7}$ N. M. Johnson, Phys. Rev. B 31, 5525 (1985).

${ }^{8}$ J. Oliva and L. M. Falicov, Phys. Rev. B 28, 7366 (1983).
${ }^{9}$ K. Muro and A. J. Sievers, Phys. Rev. Lett. 57, 897 (1986).

${ }^{10}$ G. G. DeLeo and W. B. Fowler, Phys. Rev. B 31, 6861 (1985).

${ }^{11}$ L. V. C. Assali and J. R. Leite, Phys. Rev. Lett. 55, 980 (1985); 56, 403 (1986).

${ }^{12}$ A. Amore Bonapasta, A. Lapiccirella, N. Tomassini, and M. Capizzi, Phys. Rev. B 36, 6228 (1987).

${ }^{13}$ K. J. Chang and D. J. Chadi, Phys. Rev. Lett. 60, 1422 (1988).

${ }^{14}$ J. Ihm, A. Zunger, and M. L. Cohen, J. Phys. C 12, 4409 (1979); P. J. H. Denteneer, Ph. D. thesis, Eindhoven University of Technology, 1987 (unpublished).

${ }^{15}$ Y. Bar-Yam and J. D. Joannopoulos, Phys. Rev. Lett. 52, 1129 (1984).

${ }^{16}$ C. G. Van de Walle, Y. Bar-Yam, and S. T. Pantelides, Phys. Rev. Lett. 60, 2761 (1988); C. G. Van de Walle, F. R. McFeely, and S. T. Pantelides, Phys. Rev. Lett. 61, 1867 (1988).

${ }^{17}$ E. Kaxiras and K. C. Pandey, Phys. Rev. Lett. 61, 2693 (1988).

${ }^{18}$ P. J. H. Denteneer, C. G. Van de Walle, and S. T. Pantelides, Phys. Rev. B (to be published).

${ }^{19}$ Plane waves with kinetic energy up to $6 \mathrm{Ry}$ are included exactly; those with kinetic energy between 6 and $12 \mathrm{Ry}$, in perturbation theory according to P. O. Löwdin, J. Chem. Phys. 19, 1396 (1951).

${ }^{20}$ A. D. Marwick, G. S. Oehrlein, and N. M. Johnson, Phys. Rev. B 36, 4539 (1987).

${ }^{21}$ M. Stavola, S. J. Pearton, J. Lopata, and W. C. Dautremont-Smith, Appl. Phys. Lett. 50, 1086 (1987); Phys. Rev. B 37, 8313 (1988).

${ }^{22}$ M. Stutzmann, Phys. Rev. B 35, 5921 (1987); M. Stutzmann and C. P. Herrero, Appl. Phys. Lett. 51, 1413 (1987); C. P. Herrero and M. Stutzmann, Solid State Commun. (to be published).

${ }^{23}$ M. Stavola, K. Bergman, S. J. Pearton, and J. Lopata, Phys. Rev. Lett. 61, 2786 (1988); preliminary accounts of these experimental results as well as our calculated results for the $(\mathrm{H}, \mathrm{B})$ complex were reported in Proceedings of the Fifteenth International Conference on Defects in Semiconductors, Budapest, Hungary, 1988 (to be published).

${ }^{24}$ The binding energy is defined here as the energy difference between configurations in which $\mathrm{H}$ in a specific charge state occupies the same site in $\mathrm{Ge}: \mathrm{Si}_{s}$ and in pure Ge.

${ }^{25}$ E. Merzbacher, Quantum Mechanics (Wiley, New York, 1970), p. 74. 


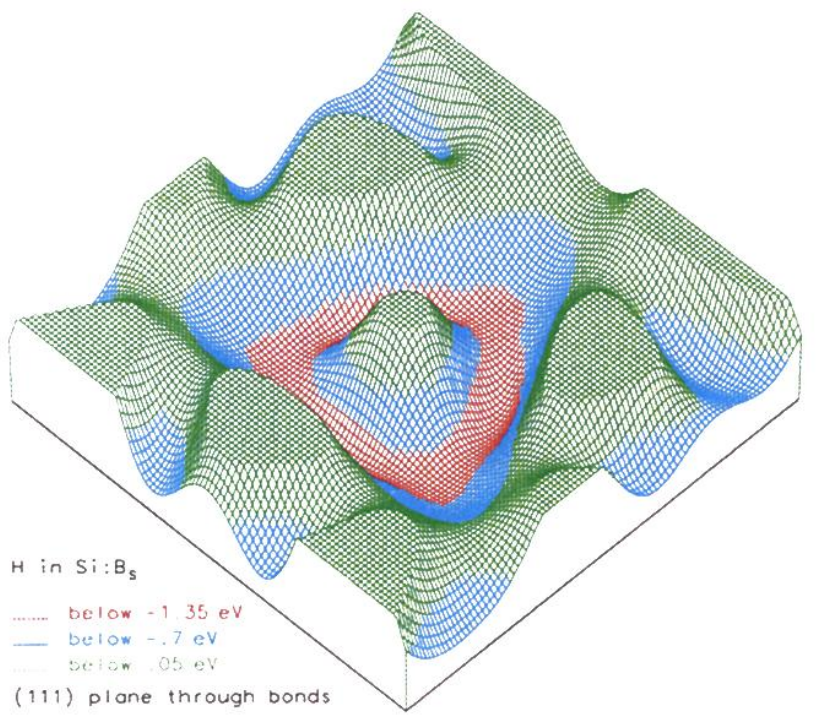

FIG. 1. Energy surface for an $\mathrm{H}$ atom in the (111) plane through three bond-minima $(B M)$ positions in $\mathrm{Si}: \mathrm{B}_{s}$. The plane does not contain atoms, but the unrelaxed lattice position of the B atom is located just $0.4 \AA$ outside the plane in the center of the red ring. The contours are color coded in three different ranges for presentation purposes. For clarity, the surface is cut off at an energy value of $0.05 \mathrm{eV}$, resulting in the green plateaus. The zero of energy is chosen at the tetrahedral interstitial site. 